\title{
Application of Neural Network and Its Extension of Derivative to Problems of Visual Realism on Web Pages
}

\author{
Lan-Ting Wang ${ }^{1,2, *}$ and Chang-Franw Lee ${ }^{1}$ \\ ${ }^{1}$ Graduate School of Design, National Yunlin University of Science and Technology, Yunlin 64002, Taiwan \\ ${ }^{2}$ Department of Visual Communication Design, Tainan University of Technology, Tainan 71002, Taiwan
}

Received: 26 Nov. 2012, Revised: 2 May. 2013, Accepted: 8 May. 2013

Published online: 1 Sep. 2013

\begin{abstract}
This study utilizes information sciences (i.e., neural networks and extensions) to reduce the burden for investigation of social sciences. Without loss of generality, the visual realism of pictures is utilized as the example to illustrate the proposed research flow chart. Pictures of web pages are important media for conveying meanings of information systems. Visual realism of pictures means the degree of similarity between pictures and real objects, and is an important topic in designing contents of information systems. In this paper, the neural network and its extension of derivative are applied to participants' preference for visual realism on web pages. This study includes three parts. In the first part, the participants' preference for visual realism on web pages is experimentally investigated. The investigation results are recorded, analyzed and discussed. In the second part, we utilize the neural network to model and predict the investigation results of the first part. With the help of neural networks, one can model and predict the full investigated results from only part of the investigated information. In the third part, we develop a neural-network extension of derivative to predict the increasing or decreasing trends of investigation data. With the help of neural-network extension of derivative, the trends of investigation data can be predicted without plotting the overall data curves. It should be noted that the neural network and its extension of derivative share the same training procedures. No additional training work is required in the neural-network extension of derivative. The use of neural network and its extension of derivative will greatly reduce the investigation efforts and one can obtain almost the same results as full investigation. This study will be helpful in understanding, modeling and predicting participants' preference for contents of information systems. Although only specific examples are illustrated in this study, the proposed research flow chart can be applied to many other problems of questionnaire investigation in social sciences.
\end{abstract}

Keywords: Neural network, neural-network extension, web pages, information system

\section{Introduction}

Due to the rapid spread of internet technologies, web pages have played important roles in our everyday life [1]. Web pages on internet are important tools of information systems, such as [2]. Through the web pages, one can access information all over the world anytime and anywhere. In designing a web page, pictures are often utilized. This is because pictures can help one easily understand the meanings communicated by displays' screens of information systems [3-5]. Visual realism for pictures of web pages is an important topic in designing the content of an information system. The visual realism of pictures means the degree of similarity between pictures and real objects [6], and is a good scale for the reality of a picture. It is impossible for a picture to completely represent a real object, i.e., there is no picture with perfect realism. In this study, the level of realism is defined to be high as a picture is very similar to the real object, and vice versa. To suitably design contents of web pages, understanding of participants' preference for visual realism of a picture is required. In general, participants' preference for visual realism of a picture is affected by their personal characteristics such as age, education, etc. This then motivates us to investigate the participants' preference for visual realism of pictures on web pages.

The main goal of this study is to utilize information sciences (i.e.,neural networks and extensions) to reduce the burden for investigation of social sciences. Without loss of generality, the visual realism of pictures is utilized as the example to illustrate the proposed research flow chart. In general, the investigation work of participants'

\footnotetext{
*Corresponding author e-mail: t40091@ mail.tut.edu.tw
} 
preference for visual realism is time consuming. This motivates us to utilize nonlinear model to predict the whole investigation data from only part of the known investigation data. There have been studies involved in modelling human behaviors by engineering approaches, such as [7]. The nonlinear model utilized in this study is the neural network. Neural networks (NN) have been widely applied to various research fields, such as [8-11]. In the past decade, we have successfully utilized neural networks to treat problems of electromagnetic waves [12-14]. In addition, we have also successfully utilized different extensions of neural networks to treat problems of antennas (extension of derivatives, [15, 16]) and sonar (extension of integration, [17]). A neural network is a network composed of many simple processors such as units, nodes and neurons $[18,19]$. One can think of the neural network as a block box (i.e., strongly nonlinear system) that accepts certain inputs and produces certain outputs. A neural network can model and predict a nonlinear system as the neural network is well trained. Since participants' preference for visual realism on web pages is strongly nonlinear and difficult to analyze, the neural network becomes a good candidate for modeling and predicting such a problem. With the help of neural networks, one can model and predict the full investigated results from only part of the investigated information. In addition to neural networks, we further develop a neural-network extension of derivative to predict the trends (i.e., increasing or decreasing) of investigation data. Such a neural-network extension of derivative has been derived and verified in our past studies $[15,16]$. By suitably extending the neural output based on the same neural-network structure, one can easily obtain the derivative of neural output with respect to neural input. It should be emphasized that no additional training work is required in the neural-network extension of derivative. The neural-network training is performed only once. That is, one can synchronously obtain the neural output and its derivative (with respect to neural input) through the same trained neural-network structure. With the help of neural-network extension of derivative, the slope information for investigation data can be predicted without plotting the overall data curves. This slope information will help us understand how the investigation data vary (e.g., increasing or decreasing). To our knowledge, this paper is the first study that applies the neural network and its extension of derivative to problems of participants' preference for visual realism of information systems. Although only specific examples are illustrated in this study, the proposed research flow chart can be applied to many other problems of questionnaire investigation in social sciences.

This study includes three parts. First, participants' preference for visual realism on web pages is experimentally investigated by questionnaires, and results are then analyzed. Second, neural networks are utilized to model and predict the investigated results in the first part. Third, a neural-network extension of derivative is developed to predict the trends (e.g., increasing or decreasing) of investigation data in the first part. The above three parts are given in Section 2, Section 3 and Section 4, respectively. Discussion is given in Section 5. Finally, the conclusion is given in Section 6.

\section{Experiments and Analyses}

The first part of this study is to experimentally investigate participants' preference for visual realism on web pages. Investigated data are statistically analyzed, and serve as the database of neural networks. The goal is to understand participants' preference for visual realism on screens of information systems. Without loss of generality, five pictures of chickens on web pages representing different levels of visual realism are utilized to test the participants, as given in Fig. 1-5. The reason why pictures of chickens are utilized is that pictures of such animals often appear in contents for science education of school's children. The five testing pictures are explained in the following.

1. Picture A (Fig. 1): This picture denotes "very high" level of visual realism, just as the photo of a real object.

2. Picture B (Fig. 2): This picture denotes "high" level of visual realism. Pictures of this type are usually drawn by hands or computer software, and are similar to photos of real objects in illumination, quality and three-dimensional shapes.

3. Picture C (Fig. 3): This picture denotes "middle" level of visual realism. Pictures of this type are usually drawn by hands or computer software, and contain main outlines and some important inner lines of real objects. However, one can not feel illumination, quality and three-dimensional perception from pictures of this type. 4. Picture D (Fig. 4): This picture denotes "low" level of visual realism. Pictures of this type are usually produced from projection, and contain only main outlines of real objects.

5. Picture E (Fig. 5): This picture denotes "very low" level of visual realism. Outlines for pictures of this type are different from those of real objects. In general, pictures of this type contain only semi-concrete or semi-discrete components. However, the fundamental characteristics of real objects can still be found in such pictures so that one can identify them.

In this part, the participants' preference among the five pictures (Fig. 1-5) is investigated. There are 210 tested participants of students and teachers from Southern Taiwan for investigation. In sampling the tested participants, the stratified and random methods are utilized for convenience, reliance and small errors. The 210 tested participants are equally divided into seven groups according to their age, as shown in Table 1. Each tested participant is asked to select his preference among the five pictures (i.e., pictures A, B, C, D, or E) in Fig. 1-5. The investigation is performed face to face. The electronic files of Fig. 1-5 are entered in a laptop computer. Each tested participant is asked to browse the 


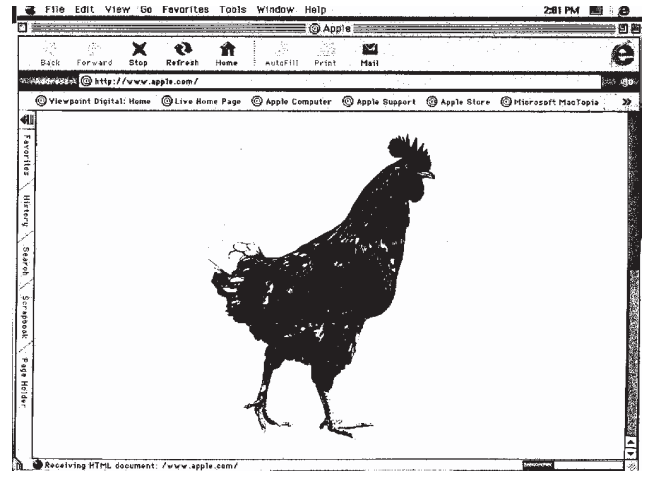

Fig. 1: Picture A: very high level of visual realism.

five pictures on the computer screen and then chooses his preference. Fig. 6 shows the percentage of tested participants in each group that prefer different pictures, i.e., different levels of visual realism. For example, for tested participants in Group \#1 (i.e., age 11-15), about $10 \%$ of them choose picture A, about $35 \%$ of them choose picture $\mathrm{B}, \ldots$, and about $12 \%$ of them choose picture E, as their preference. From Fig. 6, we can further obtain the percentage of total tested participants that prefer different levels of visual realism, as shown in Fig. 7. From Fig. 7, it shows that most of the total tested participants prefer pictures with middle or high level of visual realism. The research of reference [20] asserted that the curve of participants' preference with respect to the visual realism is similar to the Gaussian distribution. From Fig. 7, it shows that our investigation results are consistent with the points of reference [20]. Therefore, the investigated results in Fig. 6 are very reasonable.

In general, the above experimental investigation work is time-consuming. In the following two sections, the neural network and its extension of derivative are utilized to model and predict the investigation results and trends of data. We expect to predict the whole investigation data from only part of the known investigation data. The goal of such processes is to reduce the experimental investigation efforts.

Table 1 Age distribution of the total tested participants.

\begin{tabular}{cccccccc}
\hline Group & $\# 1$ & $\# 2$ & $\# 3$ & $\# 4$ & $\# 5$ & $\# 6$ & $\# 7$ \\
\hline Age & $11-$ & $16-$ & $21-$ & $26-$ & $31-$ & $36-$ & $41-$ \\
& 15 & 20 & 25 & 30 & 35 & 40 & 45 \\
\hline Participants & 30 & 30 & 30 & 30 & 30 & 30 & 30 \\
\hline \multicolumn{7}{c}{ Total: 210 participants }
\end{tabular}

\section{Neural-Network Modeling and Prediction}

The second part of this study is to utilize neural networks to model and predict the experimentally investigated results of Fig. 6. A neural network $(\mathrm{NN})$ is a network of

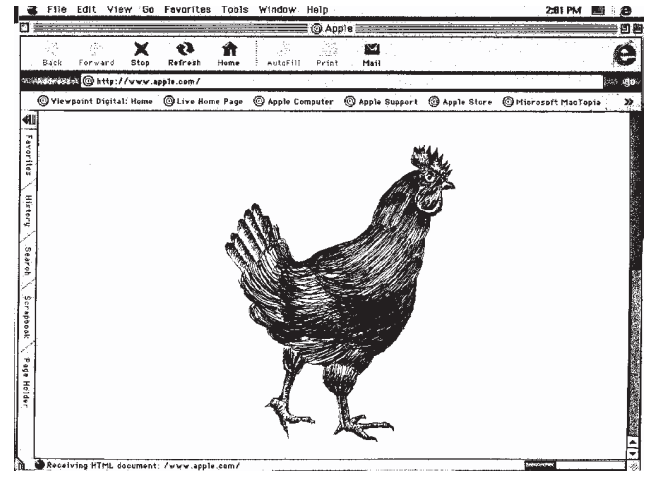

Fig. 2: Picture B: high level of visual realism.

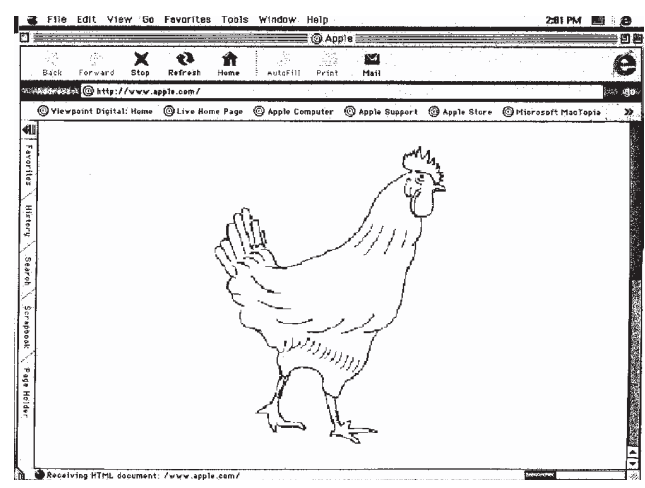

Fig. 3: Picture C: middle level of visual realism.

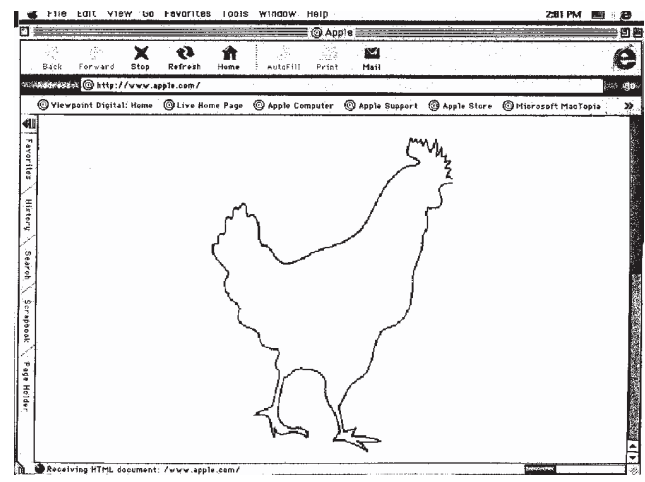

Fig. 4: Picture D: low level of visual realism.

many simple processors such as units, nodes, and neurons [18]. The processors are connected by uni-direction connections that carry numerical data. A neural network resembles the brain in two respects. One is that the knowledge is acquired by the network through a learning process. The other is that interneuron connection strengths known as synaptic weights are used to store the knowledge. For the time being, we can think of the $\mathrm{NN}$ as a black box that accepts certain inputs and 


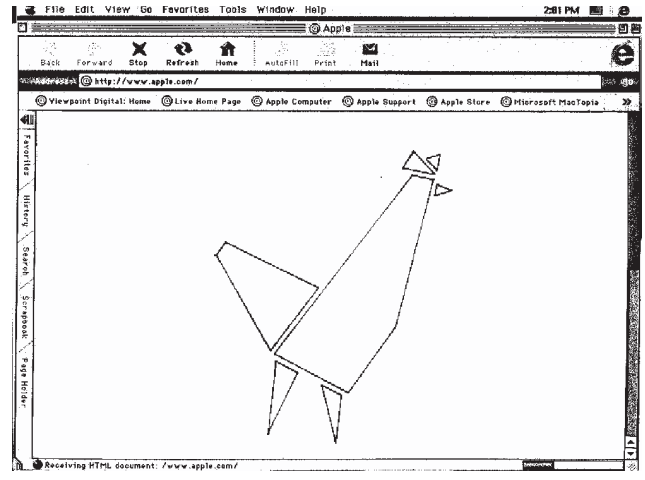

Fig. 5: Picture E: very low level of visual realism.

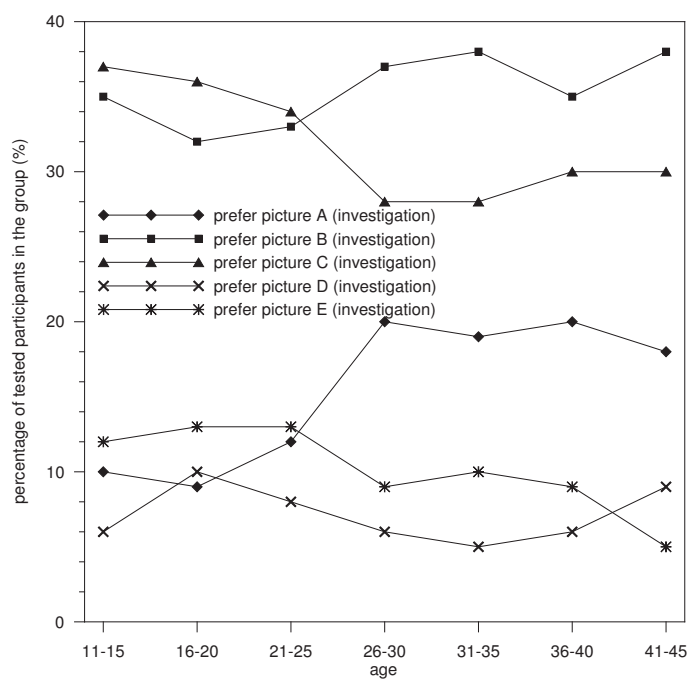

Fig. 6: the percentage of tested participants in each group that prefer different pictures, i.e., different levels of visual realism.

produces certain outputs. The functionality of the black box depends on the NN structure and the model of every neuron in this structure. An NN derives its computing power through its massively parallel distributed structure and its ability to learn and generalize (or predict). Generalization (or prediction) refers to the ability of an $\mathrm{NN}$ to provide satisfactory responses to inputs that it has not seen during its training (learning) process. The NN models process certain advantageous characteristics of nonlinearity and input-output mapping. Neural networks include different types of models. In this paper, the RBF-NN (radial basis function neural network) [19] is chosen as the model.

Consider a sophisticated system with $I$ output components $\bar{y}=\left(y_{1}, y_{2}, \ldots, y_{I}\right)$ and $K$ input components $\bar{x}=\left(x_{1}, x_{2}, \ldots, x_{K}\right)$. The analytical form for characteristics of such a sophisticated system are absent (may be unknown or even do not exist). In fact, this sophisticated system usually serves as the black-box mapping of a

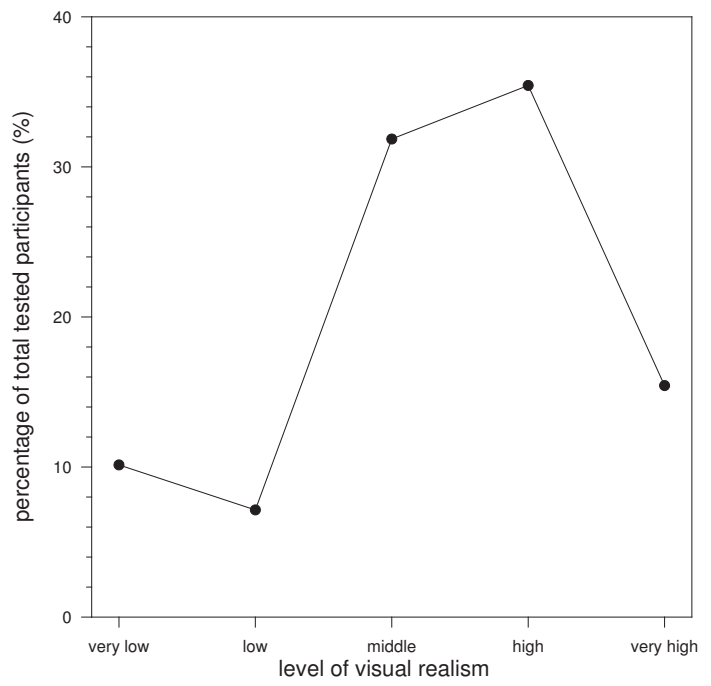

Fig. 7: The percentage of total tested participants that prefer different levels of visual realism.

complex problem, e.g., the topic of this study. The output $\bar{y}$ and the input $\bar{x}$ are mapped by the RBF-NN model. The architecture of the RBF-NN is shown in Fig. 8 (the part below the horizontal dotted line). The RBF-NN has three layers, i.e., the input layer, the hidden layer and the output layer. There are $K$ nodes in the output layer representing the output components of the system, i.e., $\bar{y}=\left(y_{1}, y_{2}, \ldots\right.$, $\left.y_{I}\right)$. There are $K$ nodes in the input layer representing the input components of the system, i.e., $\bar{x}=\left(x_{1}, x_{2}, \ldots, x_{K}\right)$. There still exist $J$ nodes in the hidden layer for nonlinear mapping. According to reference [19], the $i$-th $(i=1,2$, $\ldots, I$ ) output node of the RBF-NN is given as

$$
y_{i}=w_{i 0}+\sum_{j=1}^{J}\left[w_{i j} \cdot g_{j}(\bar{x})\right], \quad i=1,2, \ldots, I,
$$

where $w_{i j}$ and $g_{j}(\cdot)$ represent the weight and nonlinear transfer function from the $j$-th node of hidden layer toward the $i$-th node of output layer. In general, the nonlinear transfer function $g_{i j}(\cdot)$ is chosen as the Gaussian distribution and is given as [19]

$$
g_{j}(\bar{x})=\exp \left\{-\frac{\left|\bar{x}-\bar{v}_{j}\right|^{2}}{2 \sigma^{2}}\right\},
$$

where $\bar{v}_{j}$ is the mean corresponding to the $j$-th hidden node and $\sigma^{2}$ is the autocovariance of the Gaussian function. Initially, the RBF-NN is trained by known samples of $\bar{x} \rightarrow \bar{y}$. Assume the number of known samples for $\bar{x} \rightarrow \bar{y}$ is $N_{T}$. The learning strategy is described in the following.

Step 1: Select initial values for the weights from hidden to output layers. These weights are chosen to be small random values. Select initial values for the centers of the 
Gaussians in the hidden layers. These centers are randomly chosen from the training data. Select initial values for the diagonal elements of the covariances of the Gaussian functions. These variances are all chosen to be equal to some constant.

Step 2: Present the $p$-th input pattern at the input layer of the RBF-NN.

Step 3: Utilize (1) and (2) to calculate values for nodes in hidden and output layers of the RBF-NN.

Step 4: Compare the actual output $y_{i}(p)$ at the output layer

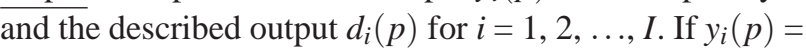
$d_{i}(p)$ for $1 \leq i \leq I$, go to Step 5. If $y_{i}(p) \neq d_{i}(p)$ for some $i$, proceed to change the weight (parameter) values by using

$$
\begin{gathered}
\Delta w_{i j}=\eta\left[d_{i}(p)-y_{i}(p)\right] g_{j}[\bar{x}(p)], \\
\Delta \bar{v}_{j}=\eta \cdot g_{j}[\bar{x}(p)] \sum_{i=1}^{I}\left[d_{i}(p)-y_{i}(p)\right] \cdot w_{i j}\left[\bar{x}(p)-\bar{v}_{j}\right],
\end{gathered}
$$

where $\eta$ denotes the learning rate.

Step 5: If $p=N_{T}$ and the cumulative error is smaller than a pre-specified threshold, we consider the training completed. If $p=N_{T}$ and the cumulative error is larger than a pre-specified threshold, then we return to Step 2 starting with the first input pattern of index $p=1$. If $p \neq$ $N_{T}$, we return to Step 2, by increasing the pattern index $p$ by one.

After the neural network is trained by some learning samples of $\bar{x} \rightarrow \bar{y}$ according to the above procedures, all the weights $w_{i j}(i=1,2, \ldots, I ; j=0,1, \ldots, J)$ will be determined. Therefore, the prediction of $\bar{x} \rightarrow \bar{y}$ will be given by (1). The trained RBF-NN can model the mapping of $\bar{x} \rightarrow \bar{y}$. In particular, the trained neural network can predict data of $\bar{x} \rightarrow \bar{y}$ that do not belong to the training data sets.

To model and predict the investigation results of Fig. 6 , parameters in the above neural-network model are selected. As shown in Fig. 8, there is one node (i.e., $x_{1}$ ) in the input layer representing the age of tested participants. In the neural computing, the seven ranges of age are encoded within the range of 0 to 1 . In our treatment, the $x_{1}$ is encoded as 1/14 for Group \#1, 3/14 for Group \#2, $\ldots$, and 13/14 for Group \#7. There are ten nodes in the hidden layer for nonlinear mapping (i.e., $J=10$ ). There are five nodes $\left(y_{1}, y_{2}, \ldots, y_{5}\right)$ in the output layer representing the percentage of tested participants that prefer pictures A, B, C, D and E, respectively. During the neural computing, values of the five output nodes are all normalized within the range of 0 to 1 . As described above, procedures of neural networks includes training and predicting stages. To illustrate the predictive power of a neural network, only part of the data in Fig. 6 are chosen for training. In our simulation, the data of Group $\# 1$, \#3, \#5, and \#7 in Fig. 6 are chosen for training the neural network. To achieve accurate neural-network prediction, such training data sets should distribute as

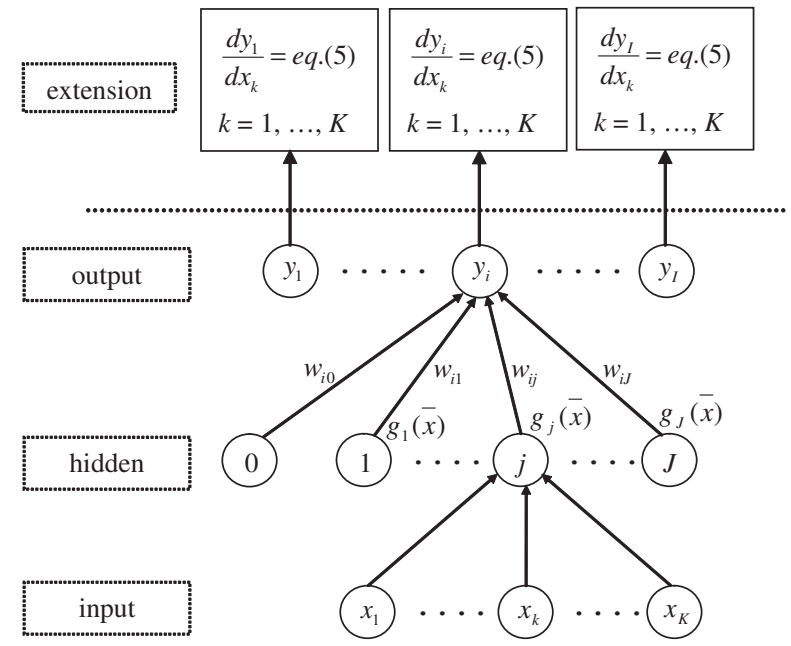

Fig. 8: Architectures for the RBF-NN and its extension of derivative.

uniform (with respect to neural input) as possible. The remaining data sets are for testing. The parameter of autocovariance in (2) is chosen as $\sigma^{2}=1 / 2$. The choices of neural-network parameters are based on experiences so that the resulting Gaussian functions are neither too peaked nor too flat [19]. In the training procedures, the learning rate $\eta$ in (3) and (4) is chosen as 0.1 and the maximum training loops are set to be $N_{T}=40000$. The training procedures have been mentioned above in details. After the neural network is trained, it can predict results beyond the training data. Fig. 9 shows the neural-network predictions for the percentage of tested participants in each group that prefer pictures representing different levels of visual realism. It shows that the neural-network prediction results (i.e., Fig. 9) are very consistent with original investigation results (i.e., Fig. 6). The average of the absolute error for data between Fig. 9 and Fig. 6 is only $0.33 \%$. That is, the RBF-NN can accurately model and predict the original investigation results. It should be noted that only part of the investigated data (i.e., training data) are required by the neural network to obtain the same results as the full investigation. From the above procedures, we conclude that the neural network can successfully model and predict behaviors of participants' preference for visual realism on web pages. Of great importance, the neural network can reduce the investigation efforts and still obtain almost the same results as the full investigation.

\section{Neural-Network Extension of Derivative}

In the third part of this paper, we develop a neural-network extension of derivative to predict the trends (e.g., increasing or decreasing) of investigation 


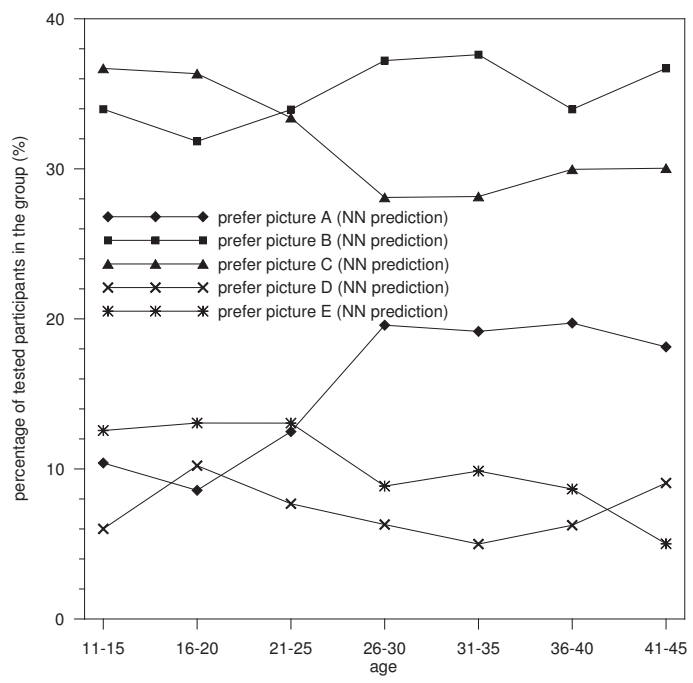

Fig. 9: Neural-network predictions for the percentage of tested participants in each group that prefer pictures representing different levels of visual realism.

data in Fig. 6. In this part, the above RBF-NN model is further extended to obtain the derivative of the output, i.e., $d y_{i} / d x_{k}(i=1,2, \ldots, I$ and $k=1,2, \ldots, K)$. For a trained neural network, the weight $w_{i j}$ of (1) is constant. From (1) and (2), we have

$$
\frac{d y_{i}}{d x_{k}}=-\frac{1}{\sigma^{2}} \sum_{j=1}^{J}\left[w_{i j}\left(x_{k}-v_{j, k}\right) \exp \left\{-\frac{\left|\bar{x}-\bar{v}_{j}\right|^{2}}{2 \sigma^{2}}\right\}\right]
$$

where $v_{j, k}$ is the $k$-th component of $\bar{v}_{j}$. Note that all the weights $w_{i j}(i=1,2, \ldots, I$ and $j=0,1, \ldots, J)$ in (5) have been determined during the learning process for establishing the model of $\bar{x} \rightarrow \bar{y}$. Therefore, a new mapping $\bar{x} \rightarrow d y_{i} / d x_{k}(i=1,2, \ldots, I$ and $k=1,2, \ldots, K)$ can be obtained from (5). In other words, the RBF-NN model can be extended to predict the derivative of the output, as shown in Fig. 8 (the part above the horizontal dotted line). It should be noted that the neural training is performed only in establishing the mapping of $\bar{x} \rightarrow \bar{y}$. No additional neural training is required in establishing the mapping of $\bar{x} \rightarrow d y_{i} / d x_{k}(i=1,2, \ldots, I$ and $k=1,2, \ldots$, $K)$. In other words, the results of $\bar{x} \rightarrow \bar{y}$ and $\bar{x} \rightarrow d y_{i} / d x_{k}$ are predicted simultaneously and the training work is performed only once.

As described in the previous section, there are five output nodes $\left(y_{1}, y_{2}, \ldots, y_{5}\right)$ and one input node $\left(x_{1}\right)$ in the RBF-NN. According to (5), $d y_{i} / d x_{k}(i=1,2, \ldots, 5)$ can be easily obtained from the trained RBF-NN. That is, the slope values for data $y_{i}(i=1,2, \ldots, 5)$ with respect to $x_{1}$ are obtained. These slope values represent properties of data distribution. A positive slope value means that the data is increasing. On the contrary, a negative slope value means that the data is decreasing. A large magnitude of slope means that the data varies violently. On the contrary, a small magnitude of slope means that the slope varies smoothly. Table 2 shows the slope information of Fig. 6 predicted from neural-network extension of derivative. From Table 2, one can give insight into the characteristics for data of Fig. 6. For example, in Table 2, the row "prefer picture A" has a slope value of 7.09 appearing at the column "age 21-25". This slope value is positive and large compared with other components in Table 2. This means that the data "prefer picture A" in Fig. 6 is going to violently increase at the point of "age 21-25". The row "prefer picture B" has a slope value of -3.64 at the column "age 31-35". This slope value is negative with large magnitude compared with other components in Table 2. This means that the data "prefer picture B" in Fig. 6 is going to violently decrease at the point of "age 31-35". The row "prefer picture E" has a slope value of -0.01 at the column "age 16-20". This slope value is very close to zero. This means that the data "prefer picture E" in Fig. 6 is almost unchanged from the point of "age 16-20". All the predictions of increase (i.e., positive slope) or decrease (i.e., negative slope) in Table 2 are consistent with practical situations in Fig. 6. It should be emphasized that no additional training work is required in using the neural-network extension of derivative. With the help of neural-network extension of derivative, the trends of investigation data can be predicted without plotting the overall data. Thus one can easily predict the trends (i.e., increasing or decreasing) of data that model participants' preference for visual realism on web pages. This will greatly reduce the efforts of practical investigation.

\section{Discussion}

Table 3 shows the relationship between roughness of sampling data and accuracy of neural-network estimation. The top 2 rows of Table 3 have the same meanings as those of Table 1, i.e., the horizontal axis of Fig. 6. In Table 3, the notation " $\sqrt{ }$ " means that the corresponding data is included in training samples of neural networks. The notation "Error" of Table 3 means the average of absolute errors for neural-network predictions. From Table 3, it shows that different selections of training samples for neural networks have different levels of predicting accuracy (i.e., different levels of predicting errors). The results of Table 3 imply that training the neural network by using more and uniformly distributed samples will have better predicting accuracy (i.e., less predicting error). This is consistent with the neural-network theories. However, too many training samples will cause more neural-computation efforts. The selection of training samples depends on experiences in corresponding research fields. In general, the number of training samples is suggested to be slightly more than half the number of whole investigation samples. 
As mentioned above, the main goal of this study is that the neural network and its extension of derivative can well model and accurately predict behaviors of participants' preference for visual realism on web pages. Note that the neural-network and its extension of derivative share the same training work. From results of Table 3, we have successfully modeled and predicted the full investigation results from only part of the investigated information. That is, the goal of this study has been achieved.

Table 2 Slope information of Fig. 6 predicted from neural-network extension of derivative.

\begin{tabular}{ccccccc}
\hline Group & $\# 1$ & $\# 2$ & $\# 3$ & $\# 4$ & $\# 5$ & $\# 6$ \\
\hline Age & $11-$ & $16-$ & $21-$ & $26-$ & $31-$ & $36-$ \\
& 15 & 20 & 25 & 30 & 35 & 40 \\
\hline Prefer A & -1.82 & 3.92 & 7.09 & -0.42 & 0.55 & -1.58 \\
\hline Prefer B & -2.14 & 2.11 & 3.27 & 0.39 & -3.64 & 2.73 \\
\hline Prefer C & -0.35 & -2.94 & -5.31 & 0.06 & 1.81 & 0.07 \\
\hline Prefer D & 4.21 & -2.54 & -1.39 & -1.30 & 1.25 & 2.82 \\
\hline Prefer E & 0.50 & -0.01 & -4.20 & 1.00 & -1.20 & -3.65 \\
\hline
\end{tabular}

Table 3 The relationship between roughness of sampling data and accuracy of neural-network estimation.

\begin{tabular}{|c|c|c|c|c|c|c|c|}
\hline $\begin{array}{c}\text { data } \\
\# 1\end{array}$ & $\begin{array}{c}\text { data } \\
\# 2\end{array}$ & $\begin{array}{c}\text { data } \\
\# 3\end{array}$ & $\begin{array}{c}\text { data } \\
\# 4\end{array}$ & $\begin{array}{c}\text { data } \\
\# 5\end{array}$ & $\begin{array}{c}\text { data } \\
\# 6\end{array}$ & $\begin{array}{c}\text { data } \\
\# 7\end{array}$ & \\
\hline Age & Age & Age & Age & Age & Age & Age & \\
\hline 11- & 16- & 21- & 26- & 31- & 36- & 41- & Error \\
\hline 15 & 20 & 25 & 30 & 35 & 40 & 45 & $\%$ \\
\hline$\sqrt{ }$ & & $\sqrt{ }$ & & $\sqrt{ }$ & & $\sqrt{ }$ & 0.33 \\
\hline$\sqrt{ }$ & & $\sqrt{ }$ & & $\sqrt{ }$ & $\sqrt{ }$ & & 0.60 \\
\hline$\sqrt{ }$ & & & $\sqrt{ }$ & & & $\sqrt{ }$ & 3.02 \\
\hline & $\sqrt{ }$ & & $\sqrt{ }$ & & $\sqrt{ }$ & & 3.51 \\
\hline & $\sqrt{ }$ & & & $\sqrt{ }$ & $\sqrt{ }$ & & 4.62 \\
\hline
\end{tabular}

\section{Conclusion}

This study has successfully utilized information sciences (i.e., neural networks and extensions) to reduce the burden for investigation of social sciences. Although only specific examples are illustrated in this study, there is no limitation on the type of questionnaire investigation. Therefore, the proposed research flow chart can be applied to many other problems of social sciences.

Without loss of generality, this study utilizes visual realism of pictures as the example to illustrate the proposed research flow chart. This is because pictures are good media for conveying meanings of information systems [21]. Good pictures can help one's understanding and communication for visions of information systems [22]. Improper pictures may disperse one' attention $[23,24]$ in watching contents of information systems. In general, pictures with high-level visual realism are only suitable in some specific environments. As the picture is too real, it will imply too many emphases and characteristics. On the contrary, an abstract picture will make one difficult to find the principal characteristics. Therefore, suitable levels of visual realism are important in designing pictures of web pages, and then in designing contents of information systems. In general, one's feeling of visual realism depends not only on the picture itself, but also on his personal characteristics (e.g., age, education, ..., etc).

In this paper, the neural network and its extension of derivative have been successfully applied to participants' preference for visual realism on web pages. Results of Section 3-5 have proven that the neural network and its extension of derivative can well model and accurately predict behaviors of participants' preference for visual realism on web pages. The RBF-NN model utilized in this study is inherently one type of the general regression [25-27]. It can model and predict strongly nonlinear problems from only part of investigation data (i.e., training data sets). Our results show that the investigated results and trends (e.g. increasing or decreasing) predicted by the neural network and its extension of derivative are very consistent with the practical investigation. Due to the use of neural networks, one can model and predict the full investigation from only part of the investigated information. With the help of neural-network extension of derivative, the trends (e.g. increasing or decreasing) of investigation data can be predicted without plotting the overall data curves. It should be noted that the neural training is performed only once, i.e., in establishing the neural-network model. In other words, the neural network and its extension of derivative share the same training procedures. No additional training work is required in the neural-network extension of derivative. The use of neural network and its extension of derivative will greatly reduce the investigation efforts, and one can obtain almost the same results as full investigation. The research flowchart of this study can also be applied to many other problems in contents of information systems.

\section{Acknowledgement}

The authors would like to acknowledge Professor Kun-Chou Lee, Department of Systems and Naval Mechatronic Engineering, National Cheng-Kung University, Taiwan, for his help in signal processing. The authors would also like to acknowledge the financial support of the National Science Council (NSC), Taiwan, under Grant NSC 99-2515-S-165-001 and NSC 99-2515-S-165-002.

\section{References}

[1] X. G. Li, Journalism \& Mass Communication Quarterly , 75, 353-365 (1998).

[2] S. M. Abbas, Ilyas Saleem, Bilal Ahmed, Hunaina Khurshid, UWB Antenna with Parasitic Patch and Asymmetric Feed, Information Sciences Letters, 2, 27-33 (2013) 
[3] M. G. Sobol, IEEE Transactions on Systems Man and Cybernetics, 19, 893-898 (1989).

[4] M. Canham and M. Hegarty, Learning and Instruction, 20, 155-166 (2010).

[5] Z. W. Zhu and Q. Ji, Machine Vision and Applications, 15, 139-148 (2004).

[6] S. M. Alessei and S. R. Trollip, Computerbased Instruction Methods and Development (2nd Ed.) (Prentice Hall, New Jersey), (1991).

[7] Z. Zainuddin and M. M. A. Shuaib, Appl. Math. Inf. Sci., 5, 53-64 (2011).

[8] A. A. Elngar, D. A. A. Mohamed, F. F. M. Ghaleb, A Real-Time Anomaly Network Intrusion Detection System with High Accuracy, Information Sciences Letters, 2, 49-56 (2013).

[9] R. K. Saeed, Iterative methods for solving nonlinear equations by using quadratic spline function, Mathematical Sciences Letters, 2, 37-43 (2013).

[10] A. B. R. Suleiman, A. Hameed, Simulation of SIPBased VoIP for Mosul University Communication Network, International Journal of Computing and Digital Systems, 2, 89-94 (2013).

[11] A. A. A. Radwan, M. H. Mohamed, M. A. Mofaddel, H. El-Sayed, A Study of Critical Transmission Range for Connectivity in Ad Hoc Network, Information Sciences Letters, 2, 77-87 (2013).

[12] K. C. Lee, Journal of Electromagnetic Waves and Applications, 17, 1217-1223 (2003).

[13] K. C. Lee, International Journal of RF and Microwave Computer-Aided Engineering, 14, 398-403 (2004).

[14] K. C. Lee, and T. N. Lin, IEEE Transactions on Antennas and Propagation, 53, 1126-1132 (2005).

[15] K. C. Lee, IEEE Transactions on Antennas and Propagation, 55, 990-993 (2007).

[16] K. C. Lee, J. Y. Jhang and T. N. Lin, IEEE Transactions on Antennas and Propagation, 57, 1270-1274 (2009).

[17] K. C. Lee, IEEE Transactions on Aerospace and Electronic Systems, 43, 1065-1070 (2007).

[18] S. Haykin, Neural networks - A Comprehensive Foundation, (Macmillan, New York, (1999).

[19] C. Christodoulous and M. Georgiopoulos, Applications of Neural Networks in Electromagnetics, (Artech House, Massachusetts,) (2001).

[20] J. A. Coey and S. Madigan, The Annual Meeting of the Western Psychological Association, Sacramento, CA, USA, May, (1975).

[21] S. J. Saunders, In: Visual Literacy, D. M. Moore and F. M. Dwyer (Ed.), 183-208, (Educational Technology Publication, New Jersey, (1994).

[22] F. M. Dwyer (Ed), Enhancing Visualize Instruction, Learning Services, Pennsylvania, (1987).

[23] L. P. Rieber, Computers, Graphics \& Learning, Brown \& Benchmark, Wisconsin, (1994).

[24] L. P. Rieber, Educational Technology Research and Development, 43, 45-46 (1995).

[25] D. F. Specht, IEEE Transactions on Neural Networks, 2, 568-576 (1991).

[26] J. Park and I. W. Sandberg, Neural Computation, 3, 246-257 (1991).

[27] J. Park and I. W. Sandberg, Neural Computation, 5, 305-316 (1993).

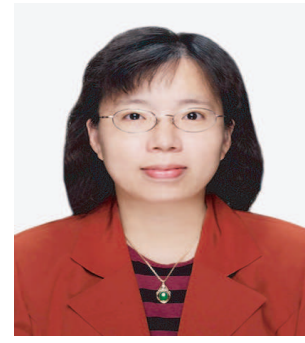

Lan-Ting Wang was born in Taiwan. She received the B.S. degree in 1989 from the Chung-Yuan University, Chung-Li, Taiwan, and M.S. degree in 1994, from the National Taiwan University of Science and Technology, Taipei, Taiwan, both in Visual Communication Design. Since 1994, she has joined the faculty of the Department of Visual Communication Design, Tainan University of Technology, Tainan, Taiwan, where she is currently an Associate Professor. Currently, she is pursuing her Ph.D. degree in the Graduate School of Design, National Yunlin University of Science and Technology, Yunlin, Taiwan. Her research interests include visual communication design, signal processing and its application to communication engineering.

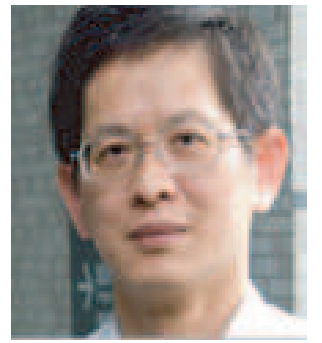

Chang-Franw Lee was born in Taiwan. He received the B.S. degree in Industrial Design from the National Cheng-Kung University, Tainan, Taiwan, and the Ph.D. degree in Nature Sciences from the Chiba University, Chiba, Japan. Currently, he is a Professor in the Graduate School of Design, and also serves as the Dean in the College of Design, National Yunlin University of Science and Technology, Yunlin, Taiwan. His research interests include green design, and the style and design of life. 